\title{
Tracheal Adenocarcinoma Treated with Adjuvant Radiation: A Case Report and Literature Review
}

\author{
Qamruzzaman Chowdhury $^{a, b} \quad$ Md. Arifur Rahman ${ }^{b} \quad$ Latifa Sultana $^{b}$ \\ M. Anwarul Islam ${ }^{\mathrm{b}}$ Golam Mohiuddin Akbar Chowdhury ${ }^{\mathrm{c}}$ \\ ${ }^{a}$ Radiation Oncology Department, National Institute of Cancer Research and Hospital, \\ ${ }^{b}$ Radiotherapy and Oncology Department, Square Hospital, and 'Thoracic Surgery, \\ National Institute of Diseases of the Chest and Hospital, Dhaka, Bangladesh
}

\section{Key Words}

Tracheal neoplasm · Adenocarcinoma · Adjuvant radiation

\begin{abstract}
Primary adenocarcinoma of the trachea is extremely rare and a standard treatment does not exist due to nonavailability of evidence-based randomized control studies. This paper reports the case of a 60-year-old male, who presented with cough and occasional respiratory distress. Bronchoscopic examination and a computed tomography scan revealed a soft tissue mass in the trachea arising from the posterior tracheal wall. Cytological examination and immunochemistry confirmed primary adenocarcinoma of the trachea. Excision of the tumor followed by three-dimensional conformal radiation therapy was performed, and a dose of 56 Gy was delivered to the primary site. Two and a half years after treatment, the patient has no clinical or radiological evidence of the disease, and no late complication has occurred.
\end{abstract}

\section{Introduction}

Primary tumors of the trachea are rare and account for $0.1 \%$ of all malignancies [1]. In adults, approximately $90 \%$ of the primary tracheal tumors are malignant, whereas in children, the majority of these tumors are benign [2,3]. In a retrospective study by Webb et al. [4], 55.4\% of the patients were male and $77.3 \%$ of them were smokers. The frequency of this rare disease has been estimated to be $0.1-0.4 \%$ of all malignancies, with an annual incidence of 2.6 new cases per million per year [5]. 
Chowdhury et al:: Tracheal Adenocarcinoma Treated with Adjuvant Radiation: A Case Report and Literature Review

Primary tracheal tumors can arise from the respiratory epithelium, salivary glands and mesenchymal structure of the trachea. Squamous cell carcinoma (SCC) and adenoid cystic carcinoma (ACC) make up about $71.6 \%$ of all adult primary tracheal tumors. The remaining portion $(28.4 \%)$ is widely distributed in a heterogeneous group of malignant tumors [4]. SCC occurs predominantly in men in the sixth and seventh decades, whereas ACC is equally distributed between the sexes and peaks in incidence in the fourth and fifth decades [6]. In contrast to ACC, SCC (the most common type of tracheal tumors) has a more aggressive course. Median survival time ranges from 6 to 14 months, and tumor disappearance is dependent on whether the primary lesion is resected. SCCs spread to the lymph nodes and $10-20 \%$ of the patients will have distant metastasis at presentation. For other pathologic variants, the clinical course and natural history are variable. Carcinoids, lymphomas, granular cell tumors and small cell carcinomas have variable prognoses; yet they seem to behave better than SCCs, adenocarcinomas or sarcomas. The majority of the tracheal neoplasms are primary in nature, and other primary sites that can metastasize to the trachea are the lung and esophagus, the latter being the most common. Patients with surgically resectable primary tracheal tumors have a better prognosis than those with tumors that cannot be resected. Preoperative radiation therapy has been attempted in some patients, yet the most compelling evidence for adjuvant radiation therapy comes from postoperative cases [7]. The present study reports a rare case of primary tracheal malignancy treated with surgery and adjuvant radiation therapy. The patient has given his informed consent and the study meets all ethical requirements.

\section{Case Presentation}

Our 60-year-old diabetic and hypertensive male patient had a 1-month history of cough associated with marked respiratory distress and occasional dyspnea in the right lateral lying position. The patient consulted a chest physician and underwent bronchoscopy, which showed a polypoid growth in the trachea extending to the larynx (fig. 1). A computed tomography (CT) scan revealed irregular soft tissue thickening in the region of the trachea extending up to the adjacent larynx with no soft tissue calcification, and there was no definite lymphadenopathy in the cervical region and no involvement of the underlying bone. A repeat CT scan of the neck (fig. 2) showed evidence of an ill-defined, irregular, nodular, infiltrating soft tissue growth arising from the posterior tracheal wall, bridging the lumen and reaching up to the anterior wall, with an infiltration of about $2.2 \times 1.8 \mathrm{~cm}$. The location was opposite the C7-D1 level involving the 5th or 6th tracheal ring, approximately $7 \mathrm{~cm}$ proximal to the carina with no significant evidence of cervical and mediastinal lymphadenopathy.

Excision of the tracheal growth through a cervical transverse incision was performed, and a tracheostomy tube was inserted. Histopathology revealed an atypical carcinoid with lymphatic invasion. Immunocytochemistry was positive for pancytokeratin and negative for chromogranin-A. The case was finally diagnosed as adenocarcinoma of the trachea.

Soon after surgery, the cough subsided and the patient attended Square Hospital, Dhaka, Bangladesh, for postoperative irradiation. The prescribed photon radiation dose was $56 \mathrm{~Gy}$ in 28 fractions. Ninety-five percent of the planning target volume (PTV) covered $97.3 \%$ of the prescribed dose. The minimum and maximum dose received by the PTV was 50.05 and 63.68 Gy, respectively. The dose distribution is illustrated below (fig. 3). Three multileaf collimator fields (left anterior oblique, right anterior oblique and posterior) were used for 
Chowdhury et al.: Tracheal Adenocarcinoma Treated with Adjuvant Radiation: A Case Report and Literature Review

three-dimensional conformal radiation therapy (3DCRT). The radiation dose distribution within the PTV is shown by color wash (fig. 3). Complete response was documented, and the patient is still alive without any evidence of disease during 30 months of follow-up.

\section{Discussion}

Primary malignant tumors of the trachea are uncommon, and therefore limited data supporting a standard management are available. The vast majority of the primary tracheal tumors in adults are malignant and most of them usually diagnosed at a later stage, which is due to a delayed presentation of specific symptoms like cough, dyspnea and hemoptysis.

It is clear that patients who can be resected have a better prognosis than those who cannot, prompting the recommendation of surgical resection for most primary tracheal tumors. All resected patients need postsurgical irradiation, regardless of tumor burden, margin status, histology or nodal status [7].

Grillo and Mathisen [8] experienced a median survival of 34 months in SCC and 118 months in ACC patients who underwent a combined modality like surgery and radiation therapy. Similarly, Chow et al. [9] attained a 61-month median survival using a combined surgery and radiation therapy in their study. Regnard et al. [10] showed that $73 \%$ of the patients with a 5-year survival underwent radiation therapy postoperatively.

The role of external beam radiation therapy as an adjuvant to surgical resection is better established. Grillo and Mathisen [8] advocated maximal (4,500-6,500 cGy) radiation therapy after SCC and ACC resection because of the close margins necessary for resection and the high likelihood of local recurrence [5]. Modern CT-based 3DCRT or intensity-modulated radiation therapy theoretically allows higher and safer doses delivered to the trachea. For postoperative cases, experiences seem to indicate that a dose of 50-60 Gy should be used. All patients with this tumor in the postoperative or definitive setting should be treated with the 3DCRT technique. An intraluminal boost technique after external beam radiation therapy may decrease the risk of late side effects [7].

There are many studies showing the benefit of postoperative irradiation therapy in primary tracheal malignancies such as SCCs and ACCs. However, little experience has been shared in the case of primary adenocarcinomas of the trachea, the tumor type described in our report. The prognosis of patients with malignant tumors of the trachea remains gloomy, and long-term median survival of tracheal adenocarcinoma patients undergoing combined modalities is unknown to us. Primary adenocarcinoma of the trachea needs to be diagnosed at an early stage, and combined multimodal approaches may be explored to attain an extended median survival.

\section{Disclosure Statement}

None of the authors has any conflicts of interest regarding the content of this article.

\section{References}

1 Papadopoulou A, Froudarakis M, Abatzoglou I, Koukourakis MI: Tracheal cancer treated with a short course of external and endoluminal radio-chemotherapy combined with cetuximab - a case report. J Contemp Brachyther 2010;2:160-162.

2 Culp OS: Primary carcinoma of trachea. J Thorac Cardiovasc Surg 1938;7:471-478. 


\section{Case Reports in Oncology}

\begin{tabular}{l|l}
\hline Case Rep Oncol 2013;6:280-284 & \\
\hline DOI: $10.1159 / 000351736$ & $\begin{array}{l}\text { C) 2013 S. Karger AG, Basel } \\
\text { www.karger.com/cro }\end{array}$ \\
\hline
\end{tabular}

Chowdhury et al.: Tracheal Adenocarcinoma Treated with Adjuvant Radiation: A Case Report and Literature Review

-3 Ampil FL: Primary malignant tracheal neoplasms: case reports and literature radiotherapy review. J Surg Oncol 1986;33:20-23.

4 Webb BD, Walsh GL, Roberts DB, Sturgis EM: Primary tracheal malignant neoplasms: the University of Texas MD Anderson Cancer Center experience. J Am Coll Surg 2006;202:237-246.

5 Meyers FB, Mathisen DJ: Management of tracheal neoplasm. Oncologist 1997;2:245-253.

6 Compeau CG, Keshavvee S: Management of tracheal neoplasm. Oncologist 1996;1:347-353.

7 Perez CA, Brady LW, Halaexin EC, Schmidt-Ullrich RK: Principles and Practice of Radiation Oncology, ed 4. Philadelphia, Lippincott Williams \& Wilkins, 2004, pp 1265-1268.

8 Grillo HC, Mathisen DJ: Primary tracheal tumors: treatment and results. Ann Thorac Surg 1990;49:69-70.

-9 Chow DC, Komaki R, Libshitz HI, et al: Treatment of primary neoplasms of the trachea. The role of radiation therapy. Cancer 1993;71:2946-2952.

$\checkmark 10$ Regnard JF, Fourquier P, Levasseur P: Results and prognostic factors in resections of primary tracheal tumors: a multicenter prospective study. The French Society of Cardiovascular Surgery. J Thorac Cardiovasc Surg 1996;111:808-813.

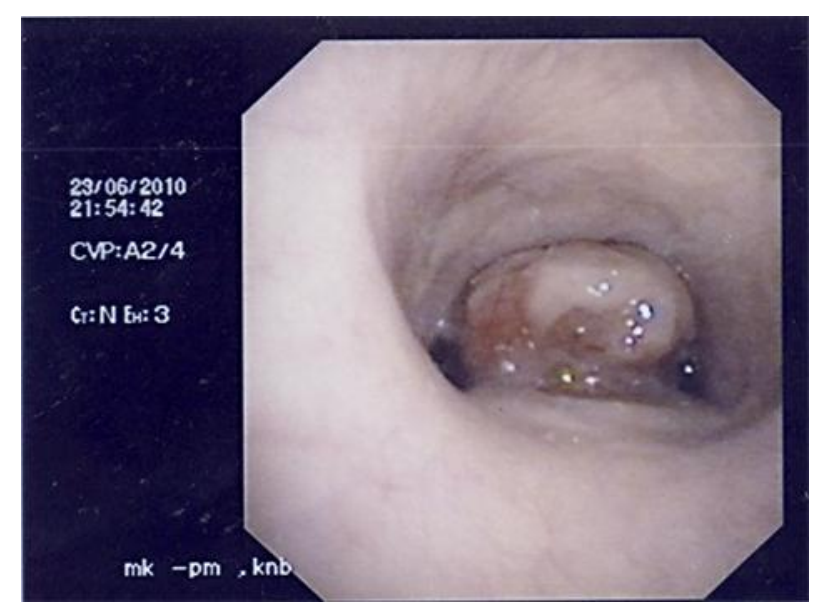

Fig. 1. Bronchoscopic examination reveals a polypoid growth in the trachea. 


\section{Case Reports in Oncology}

\begin{tabular}{l|l}
\hline Case Rep Oncol 2013;6:280-284 & \\
\hline DOI: $10.1159 / 000351736$ & $\begin{array}{l}\text { C 2013 S. Karger AG, Basel } \\
\text { www.karger.com/cro }\end{array}$ \\
\hline
\end{tabular}

Chowdhury et al.: Tracheal Adenocarcinoma Treated with Adjuvant Radiation: A Case

Report and Literature Review

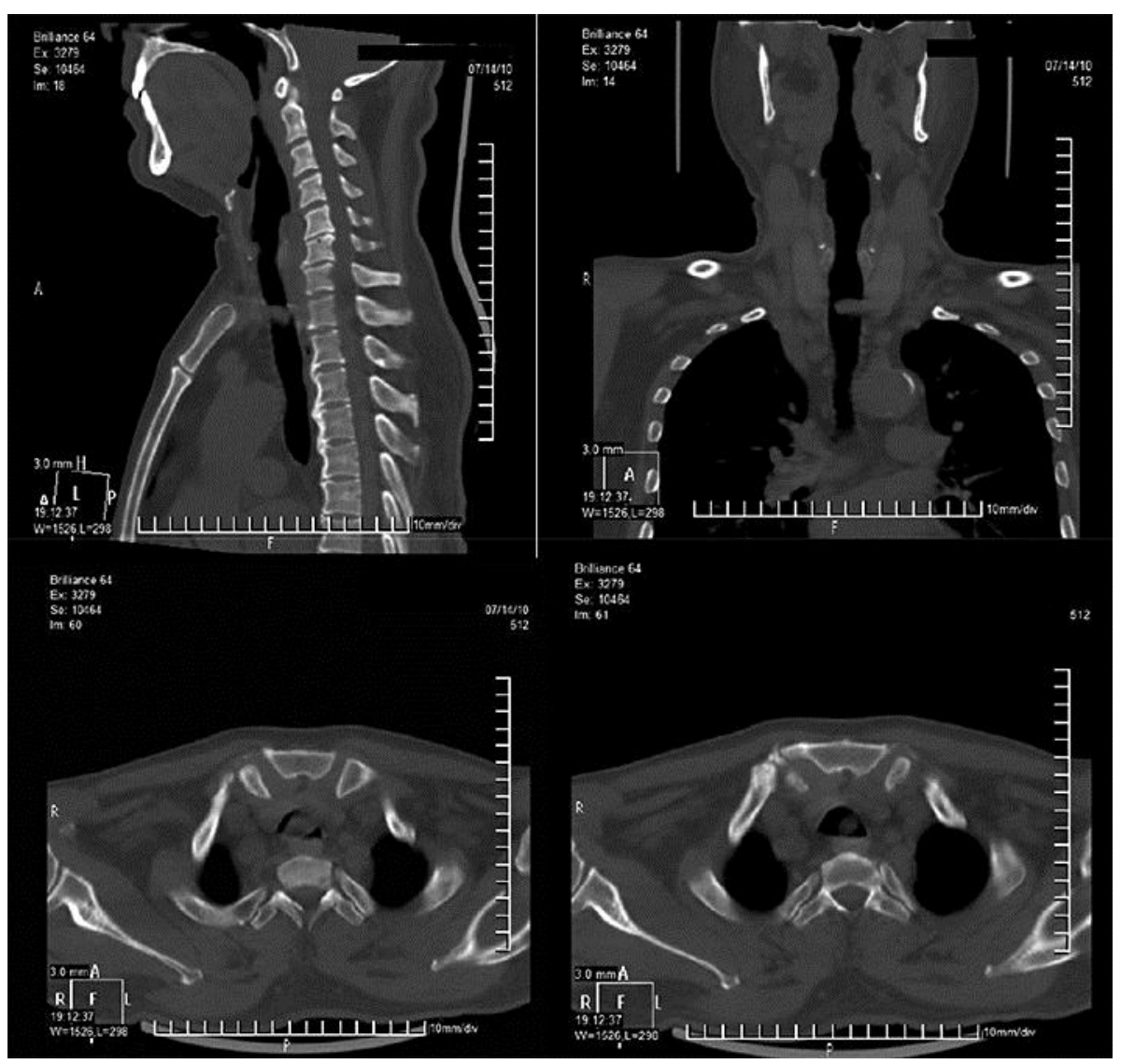

Fig. 2. A CT scan reveals a soft tissue growth arising from the posterior tracheal wall.
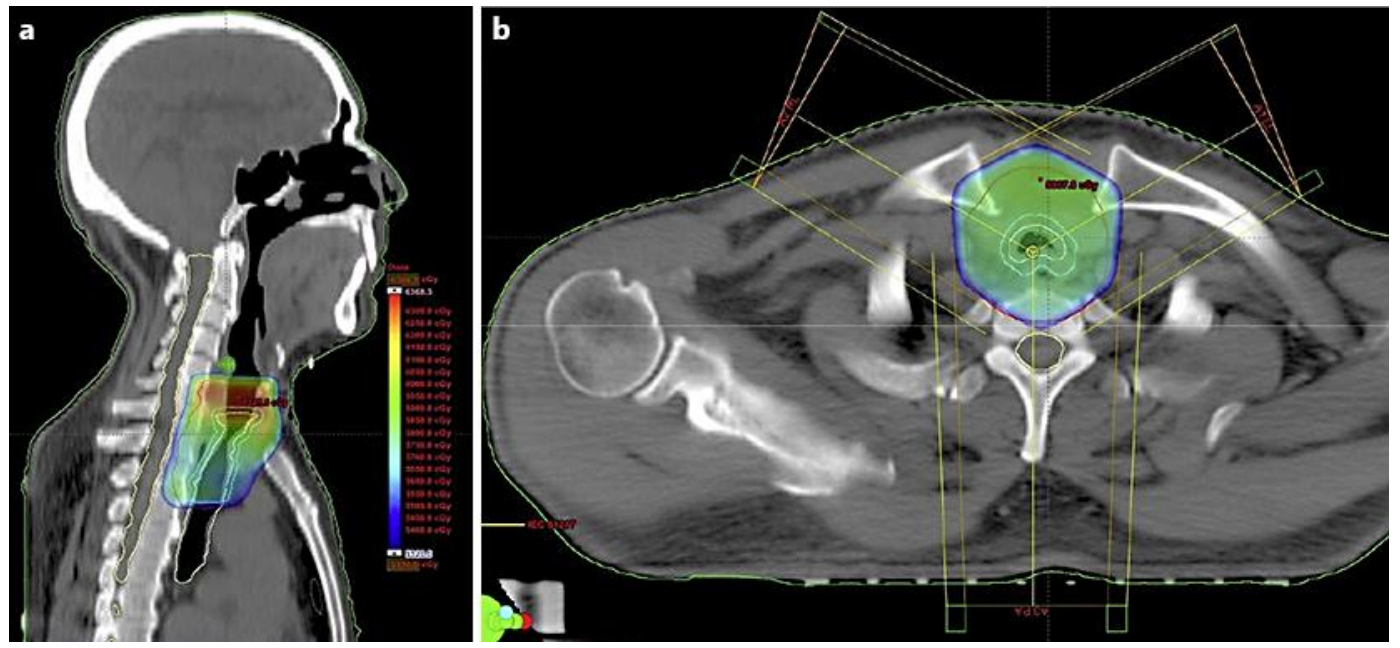

Fig. 3. a Sagittal view and $\mathbf{b}$ transverse view of the dose distribution of radiation treatment. 